\title{
Case Report: De Quervain's Thyroiditis as a Long-Term Sequelae Complication to SARS-CoV-2 Infection
}

\author{
Mark Kong ${ }^{a}$ Sarah La Porte ${ }^{b}$ \\ aDepartment of Radiology, Oxford University Hospitals NHS Trust, Oxford, UK; ${ }^{b}$ Department \\ of Radiology, Milton Keynes University Hospitals NHS Trust, Milton Keynes, UK
}

\section{Keywords}

De Quervain's thyroiditis · Long-term complication · SARS-CoV-2 · Thyroid ultrasound ·

Critical care

\begin{abstract}
A 44-year-old man presented with an enlarged painful lower anterior neck lump with elevated serum concentrations of free thyroxine (T4) and tri-iodothyronine (T3), alongside the presence of antithyroid peroxidase antibodies. Prior to presentation, the patient was demonstrating recovery from a SARS-CoV-2 infection that required sedation, intubation, and invasive ventilation in the intensive care unit (ICU) for 11 days. Ultrasound examination of the thyroid demonstrated features of De Quervain's (subacute) thyroiditis. This corresponded to the clinical picture, and continuous thyroid function tests were arranged. Emerging evidence throughout the SARS-CoV-2 pandemic describes the long-term sequelae of the infection, including developing atypical effects on the thyroid gland. This case report emphasises the association of painful subacute thyroiditis with post-viral infection and its manifestation during recovery from severe SARS-CoV-2, suggesting that follow-up thyroid function testing should be considered in patients discharged from the ICU who develop neck discomfort.
\end{abstract}

(C) 2021 The Author(s).

Published by S. Karger AG, Basel

\section{Introduction}

Subacute thyroiditis is a self-limiting inflammatory disorder of the thyroid gland first described by Swiss surgeon De Quervain in 1902 [1]. Characterised by systemic symptoms of myalgia, malaise, and fatigue, this is accompanied with severe anterior neck pain and swelling of the thyroid gland. Although an uncommon condition, with an overall incidence of approximately 12.1 per 100,000 per year, De Quervain's thyroiditis is the most common cause of 
thyroid pain and is widely considered to occur as a post-viral inflammatory response to a recent respiratory viral infection [2].

Initial thyroid function tests demonstrate hyperthyroidism, which can last several weeks, with a subsequent hypothyroid picture, which persists for several months, before normalising in approximately $95 \%$ of patients [3]. Further hallmarks include an elevated serum concentration of C-reactive protein, erythrocyte sedimentation rate, and leucocyte counts.

The diagnosis of thyroiditis is usually based upon clinical grounds, with thyroid function testing and ultrasound for confirmation. Fine-needle aspiration of the thyroid will also demonstrate cytological appearances consistent with a granulomatous thyroiditis [4]. As the UK recovers from its second peak of infections, with total cases approaching 4.5 million $[5,6]$, the long-term impacts of this viral infection will arise over the coming months, and especially for those who are admitted for ventilatory support.

This case report describes an intensive care unit (ICU) admission during the first peak of the coronavirus pandemic in March 2020 for a patient who required intubation and ventilation after developing severe SARS-CoV-2 pneumonitis. After successful extubation and an extended recovery, the patient developed severe anterior neck pain and swelling that clinically corresponded to a diagnosis of De Quervain's thyroiditis that was confirmed biochemically and with ultrasound.

\section{Case Report}

A 44-year-old man initially presented to the emergency department with shortness of breath, loose stools, fatigue, and hypoxia with oxygen saturations of $84 \%$ on air. The patient was a nonsmoker with no history of previous lung disease, no recent travel abroad, or known contacts with SARS-CoV-2 positivity. His past medical history consisted of hypertension, long-standing chronic glomerulonephritis, and stage III chronic kidney disease (stable eGFR of 30-35). He had no known allergies, and his long-term medications included amlodipine, doxazosin, and ramipril for chronic renal disease-related hypertension.

On initial admission, the patient was alert, febrile $\left(39.4^{\circ} \mathrm{C}\right)$, and dyspnoeic with a respiratory rate of $33 / \mathrm{min}$ and an oxygen saturation of $84 \%$ on room air. He was placed on $15 \mathrm{~L}$ of oxygen therapy, a septic screen was performed, intravenous antibiotics and fluids were given alongside urinary output monitoring. Initial chest radiographs demonstrated patchy consolidation at the left lung base (Fig. 1). A critical care review found an oxygen saturation of $92 \%$ on 15L of oxygen with fatigue and a deteriorating work of breathing. After further deterioration, the decision was made to proceed with sedation, intubation and ventilation using an emergency transfer ventilator in resus before being transferred to the ICU for long term mechanical ventilation. This was discussed and agreed with the patient and his family.

The patient remained sedated and ventilated for 11 days. A repeat chest radiograph demonstrated progression of the pulmonary opacification, bilaterally affecting both lung bases (Fig. 1). The patient's oxygen requirement peaked at day 4 before hypoxaemia began to improve and slow weaning commenced.

Respiratory viral PCR was positive for the SARS-CoV-2 virus. The patient was negative for adenovirus, influenza A and B, parainfluenza viruses, rhinovirus, and human enterovirus, alongside negative urinary pneumococcal and legionella antigen testing. Blood-borne virus and sickle cell screening was also negative.

The patient was successfully extubated, requiring a slow wean off oxygen therapy to room air, and recovery with regular physiotherapy for a further 7 days until discharge. The post-extubation chest radiograph demonstrated less extensive, residual patches of airspace opacification throughout both lung fields.

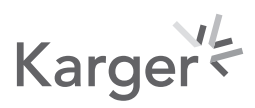



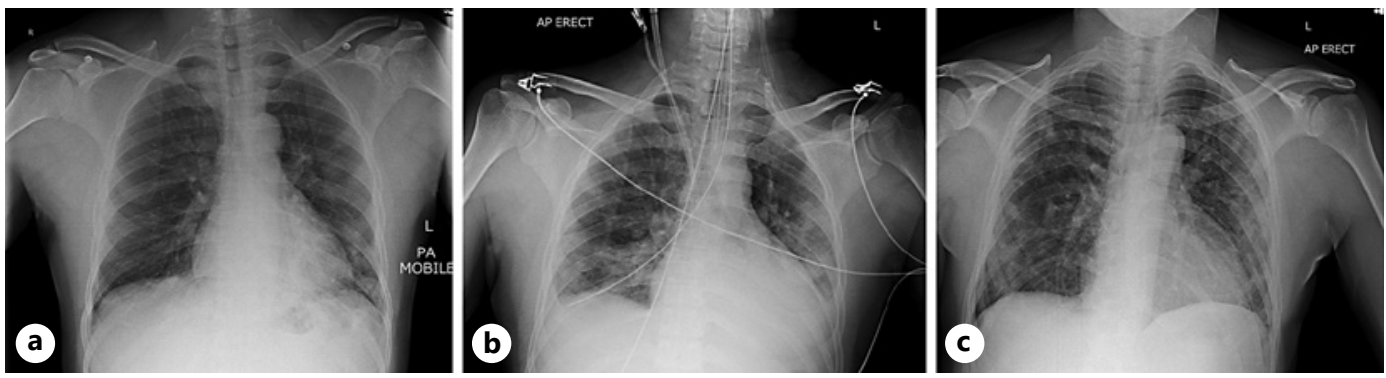

Fig. 1. Chest radiographs during admission for SARS-CoV-2 infection. These chest radiographs demonstrated the progression of airspace opacification from the initial presentation in the emergency department (a) to being placed on invasive ventilation support within ICU (b) and the patient upon extubation (c). ICU, intensive care unit.

Once discharged, the patient continued his recovery at home with follow-up outpatient respiratory physiotherapy consultations over the telephone. The patient described persistent exercise limiting dyspnoea after discharge and he was unable to return to functional baseline for 5 months. After discharge, the patient began to develop significant malaise and odynophagia, and after 2 weeks, he further developed anterior neck swelling and persisting pain. There was no intervening illness.

Due to general worsening of anterior neck pain with no improvement, a 10-day course of oral penicillin antibiotics was completed; he presented again with a significant painful goitre, with pain on swallowing, and no signs of infection on examination. He described the pain initially on the right side but eventually moving to the contralateral side of the anterior neck. The patient further described some signs of thyrotoxicosis, including slight weight loss, general malaise, and mild autonomic symptoms of heat intolerance. There was no evidence of tremor, palpitations, or anxiety, though an increased resting heart rate of $90 \mathrm{bpm}$ was noted.

Blood tests demonstrated a hyperthyroid picture with positivity for antithyroid peroxidase antibodies and raised inflammatory markers (Table 1). After receiving advice from the hospital endocrinologist, he was prescribed propranolol $40 \mathrm{mg}$ once daily for symptom relief with follow-up thyroid function testing and an appointment for ultrasound imaging of the neck.

\section{Ultrasound Features}

The features on the neck ultrasound examination were characteristic of subacute thyroiditis. Geographic hypoechoic areas predominantly seen in the anterior aspects of both enlarged thyroid lobes demonstrated minimal colour flow Doppler signal (Fig. 2). Surrounding reactive cervical lymph nodes were seen, with normal appearance of the salivary glands.

Due to the characteristic image pattern, the use of fine-needle aspiration was not required. As no thyroid nodules were present that were suspected of causing the hyperthyroid picture, the use of radionuclide thyroid imaging would also be limited and would generally demonstrate poor uptake of the radiotracer in painful subacute thyroiditis [7]. Therefore, in the clinical setting, the ultrasound features alongside the biochemical profile of hyperthyroidism and raised inflammatory markers are distinctive for De Quervain's thyroiditis, which in this case has arisen during the recovery for severe SARS-CoV-2 viral infection.

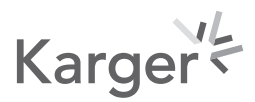


Table 1. Relevant blood tests on presentation with anterior neck pain and swelling

\begin{tabular}{llll}
\hline Blood test & Results & Normal range & Units \\
\hline TSH & 0.009 & $0.38-5.33$ & $\mathrm{miu} / \mathrm{L}$ \\
Thyroxine (T4) & 26.3 & $7-16$ & $\mathrm{pmol} / \mathrm{L}$ \\
Tri-iodothronine (T3) & 7.4 & $3.8-6$ & $\mathrm{pmol} / \mathrm{L}$ \\
TPOAb & Positive & & $\rightarrow$ \\
TSH receptor antibodies & $<0.3$ & $0-1.75$ & $\mathrm{IU} / \mathrm{L}$ \\
Serum immunoglobulins & & & \\
$\quad$ IgA & 4.5 & $0.8-2.8$ & $\mathrm{~g} / \mathrm{L}$ \\
$\quad$ IgG & 19.7 & $6-16$ & $\mathrm{~g} / \mathrm{L}$ \\
$\quad$ IgM & 0.7 & $0.5-1.9$ & $\mathrm{~g} / \mathrm{L}$ \\
C-reactive protein & 171 & $0-6.0$ & $\mathrm{mg} / \mathrm{L}$ \\
Erythrocyte sedimentation rate & 104 & $1-10$ & $\mathrm{~mm} / \mathrm{h}$ \\
Neutrophils & 9.7 & $1.7-7.5$ & $\times 10^{9} / \mathrm{L}$ \\
\hline
\end{tabular}

TSH, thyroid-secreting hormone; TPOAb, antithyroid peroxidase antibodies.
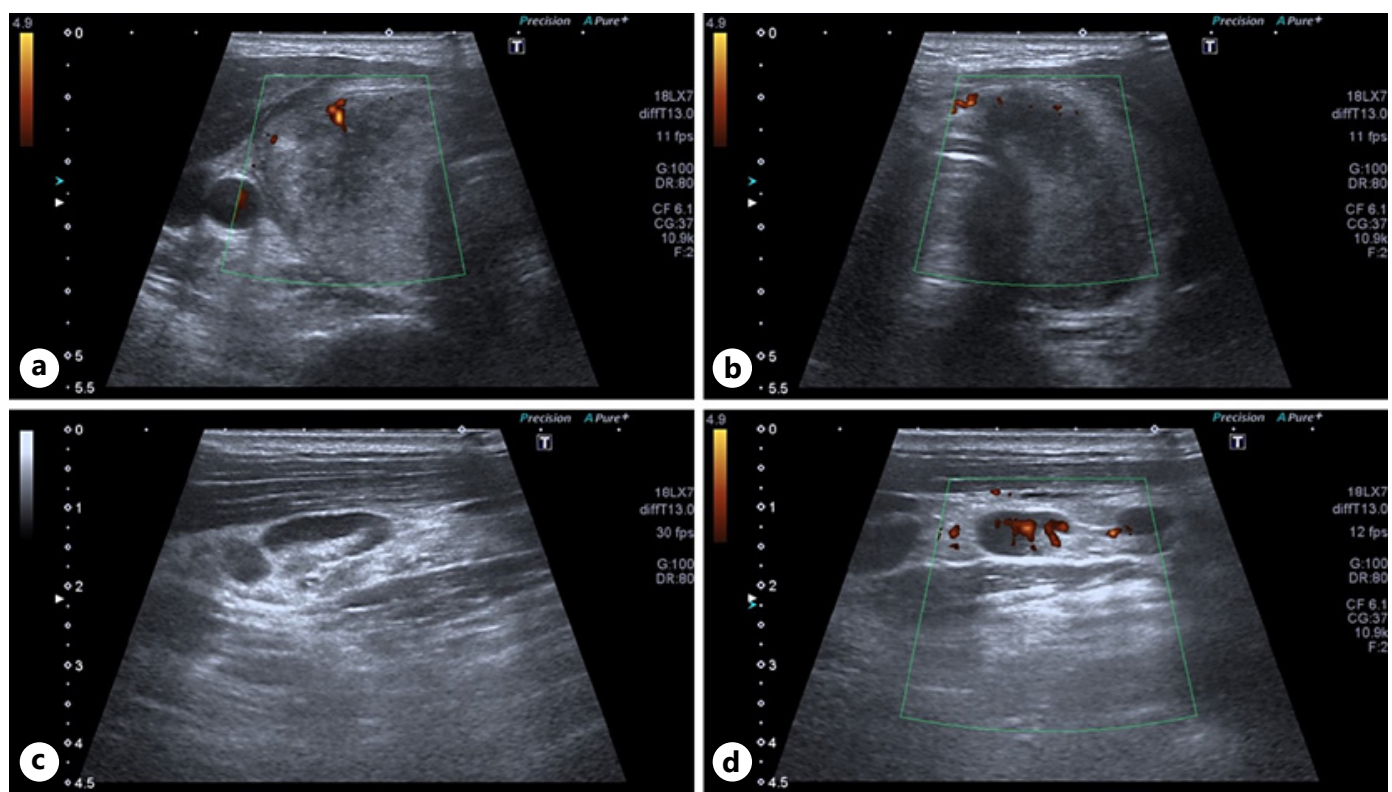

Fig. 2. Ultrasound thyroid examination. The findings of the ultrasound examination demonstrated ill-defined geographic regions of hypoechogenicity distributed across the gland with minimal vascularity. This was bilateral as seen in the right thyroid lobe (a) and left thyroid lobe (b). The gland was diffusely enlarged with no visible thyroid nodules. Enlarged cervical neck lymph nodes in levels II and III were also demonstrated (c, d), with benign appearances of fatty hilum, ovoid shape, and normal vascular pattern. Findings were consistent with De Quervain's/Subacute thyroiditis.

\section{Outcome and Treatment}

The patient was commenced on $40 \mathrm{mg}$ propranolol once daily and simple analgesia, including paracetamol and nonsteroidal anti-inflammatory drug (ibuprofen) for symptom management. At 3 weeks after presentation, a hyperthyroid biochemical picture persisted 
(thyroid-secreting hormone [TSH] $0.006 \mathrm{mIU} / \mathrm{L}$, free T4 $21.7 \mathrm{pmol} / \mathrm{L}$, and free T3 5.2 $\mathrm{pmol} / \mathrm{L}$ ). The anterior neck pain settled after approximately 6 weeks since symptoms were first detected, with the swelling and pain of the goitre similarly reducing over this time period. The symptoms of thyrotoxicosis had also improved, with overall resolution of general malaise and hot flushes. As the symptoms had settled, the use of corticosteroids for pain relief was considered unnecessary, although this is an option considered effective in relieving severe neck pain as a result of thyroiditis [8]. As thyrotoxicosis in subacute thyroiditis is due to the release of preformed T3 and T4, the use of antithyroid medications is not considered effective in reducing the serum concentrations of these hormones.

Repeat serum TSH concentration at 8 weeks after presentation demonstrated a mild hypothyroid biochemical picture (5.22 $\mathrm{mIU} / \mathrm{L}$, normal range $0.38-5.33 \mathrm{mIU} / \mathrm{L})$. This hypothyroid period lasted a further 4 weeks before normalisation of thyroid hormones.

\section{Discussion/Conclusion}

This report adds to the emerging evidence of the SARS-CoV-2 association with subacute thyroiditis and details the first patient in the UK requiring invasive ventilation for SARSCoV-2 pneumonitis to develop subacute thyroiditis during recovery. Reports of thyroiditis have been described in patients with mild symptoms of the SARS-CoV-2 virus [9-12]. However, some evidence has also demonstrated that higher rates of hyperthyroidism or low TSH concentrations are measured in SARS-CoV-2 patients during ICU admission, and this may further predispose them to develop thyroiditis $[13,14]$.

In this case, the patient did have one normal serum TSH concentration taken on day 2 of intubation, measuring 1.4 (normal range $0.38-5.33$ ) $\mathrm{mIU} / \mathrm{L}$. The onset of subacute thyroiditis did not appear in the days after extubation but once the patient approached clinical resolution of the infection weeks after discharge. In a prior case series of patients with mild infection, all demonstrated the onset of subacute thyroiditis between 16 and 36 days after clinical resolution of SARS-CoV-2 infection [12]. Hence, the onset may be delayed further in patients severely affected by the virus who require mechanical ventilation, who take significantly longer to reach clinical resolution and therefore may develop subacute thyroiditis at a later stage.

Various common viral pathogens have been epidemiologically linked to developing subacute thyroiditis. These include influenza, adenovirus, echovirus, mumps, coxsackie, and H1N1 viruses that can demonstrate elevated post-convalescent viral titres during the onset of subacute thyroiditis [15]. The propensity of different viral agents to affect the thyroid is also suggested by the high prevalence in the seasonal distribution after viral outbreaks. While cases of subacute thyroiditis were not described as a result of the first coronavirus outbreak of SARS-CoV-1, reports of thyroid complications typically involved post-viral hypothyroidism [16]. Therefore, SARS-CoV-2 appears to possibly demonstrate a greater association with subacute thyroiditis than SARS-CoV-1.

A number of pathophysiological mechanisms have been proposed that include local inflammatory responses from systemic SARS-CoV-2 infection yielding apoptosis of thyroid tissue, direct viral replication within the thyroid gland, transient hypothalamicpituitary-thyroid axis dysfunction, and interactions with the angiotensin-converting enzyme 2 (ACE-2) receptor $[17,18]$. Although the exact mechanism of thyroid dysfunction has not yet been fully established, increasing evidence suggests that direct infection of the thyroid gland may be the primary cause [18]. In particular, the ACE-2 receptor is considered a key entry mechanism by the $\mathrm{S} 1$ subunit on the spike protein of the SARS-CoV-2

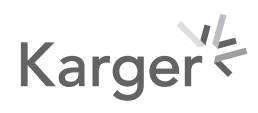


virus for lung damage, and this entry pathway exhibits greater expression in thyroid cells than in the lung tissue $[19,20]$. This was confirmed with the detection of encoding mRNA within thyroid follicular cells for ACE- 2 and the TMPRSS2 protease that cleaves the spike protein to produce the S1 subunit for receptor binding [21, 22]. Furthermore, extensive follicular apoptosis was described in autopsy studies of the SARS-CoV-1 virus to support the likely induction of direct thyroid gland damage [23]. This would also be consistent with a resulting anamnestic autoimmune response that may then theoretically arise in response to the thyroid gland as a result of viral and host cell membrane antigen complexes targeted by cytolytic T-cell recognition. Whether thyroiditis arises from the direct infection of the gland itself, the immune response towards the virus, or a combination of these mechanisms remains unclear. Though, with the onset of thyroiditis shortly after direct viral symptoms resolve, it is highly likely that the adaptive immune response plays a significant role.

The possibility of coincidental thyroiditis cannot be excluded in this individual due to the high prevalence of confirmed SARS-CoV-2 infections. However, as there was no intervening illness, SARS-CoV-2 remains the most likely precipitating factor. Thus, in the case of a viral pandemic where large numbers are documented, epidemiological study should lead to increasing numbers of reports as the late sequelae of SARS-CoV-2 infection emerge. This has been seen to some extent, with rising reports of De Quervain's thyroiditis across the globe. Therefore, with the increasing numbers of patients recovering from mechanical airway ventilation, clinicians should be made aware of this potentially delayed post-viral complication and suggests that thyroid function testing should be considered in patients recovering from SARS-CoV-2 who develop neck discomfort.

\section{Statement of Ethics}

The patient gave their written informed consent for publication of the details of their medical case and any accompanying images. All attempts have been made to make the case anonymous and nonidentifiable. This report is exempt from ethical committee approval as per the UK National Service research governance arrangements. This case report was completed in accordance with the Helsinki Declaration as revised in 2013.

\section{Conflict of Interest Statement}

The authors have no conflicts of interest to declare.

\section{Funding Sources}

No funding was received.

\section{Author Contributions}

M.K. gathered the clinical information and wrote the initial draft. S.L.P. edited subsequent drafts. The final draft was agreed upon by both authors. 


\section{References}

1 Quervain F. Über acute, nichteitrige thyroiditis. Arch Für Klin Chir. 1902;67:706-14.

2 Golden SH, Robinson KA, Saldanha I, Anton B, Ladenson PW. Prevalence and incidence of endocrine and metabolic disorders in the United States: a comprehensive review. J Clin Endocrinol Metab. 2009 Jun 1;94(6): 1853-78.

3 Pearce EN, Farwell AP, Braverman LE. Thyroiditis. N Engl J Med. 2003 Jun 26;348(26):2646-55.

4 García Solano J, Giménez Bascuñana A, Sola Pérez J, Campos Fernández J, Martínez Parra D, Sánchez Sánchez C, et al. Fine-needle aspiration of subacute granulomatous thyroiditis (De Quervain's thyroiditis): a clinicocytologic review of 36 cases. Diagn Cytopathol. 1997 Mar;16(3):214-20.

5 Dong E, Du H, Gardner L. An interactive web-based dashboard to track COVID-19 in real time. Lancet Infect Dis. 2020 May 1;20(5):533-4.

6 Public Health England. Official UK coronavirus dashboard [Internet]. Official UK coronavirus dashboard, patients in mechanical ventilation beds. [cited 2021 Jan 31]. Available from: https://coronavirus.data.gov.uk/ details/healthcare.

7 Daniels GH, Li JH, Barbesino G. Imaging “thyroiditis”: a primer for radiologists. Curr Probl Diagn Radiol. 2020 Sep.

8 Volpé R. The management of subacute (DeQuervain's) thyroiditis. Thyroid. 1993 Jan;3(3):253-5.

9 Mattar SAM, Koh SJQ, Rama Chandran S, Cherng BPZ. Subacute thyroiditis associated with COVID-19. BMJ Case Rep. 2020 Aug;13(8):e237336.

10 Chakraborty U, Ghosh S, Chandra A, Ray AK. Subacute thyroiditis as a presenting manifestation of COVID-19: a report of an exceedingly rare clinical entity. BMJ Case Rep. 2020 Dec;13(12):e239953.

11 Khatri A, Charlap E, Kim A. Subacute thyroiditis from COVID-19 infection: a case report and review of literature. Eur Thyroid J. 2020 Nov 5:1-5.

12 Brancatella A, Ricci D, Cappellani D, Viola N, Sgrò D, Santini F, et al. Is subacute thyroiditis an underestimated manifestation of SARS-CoV-2 infection? Insights from a case series. J Clin Endocrinol Metab. 2020 Oct 1; 105(10):e3742-6.

13 Kim BW. Critically Ill COVID-19 patients may be prone to subacute thyroiditis. Clin Thyroidol. 2020 Sep 1; 32(9):412-4.

14 Muller I, Cannavaro D, Dazzi D, Covelli D, Mantovani G, Muscatello A, et al. SARS-CoV-2-related atypical thyroiditis. Lancet Diabetes Endocrinol. 2020 Sep;8(9):739-41.

15 Desailloud R, Hober D. Viruses and thyroiditis: an update. Virol J. 2009;6(1):5.

16 Chen W, Tian Y, Li Z, Zhu J, Wei T, Lei J. Potential interaction between SARS-CoV-2 and thyroid: a review. Endocrinology. 2021 Jan 11;162:bqab004.

17 Scappaticcio L, Pitoia F, Esposito K, Piccardo A, Trimboli P. Impact of COVID-19 on the thyroid gland: an update. Rev Endocr Metab Disord. 2020 Nov 25. http://link.springer.com/10.1007/s11154-020-09615-z.

18 Aemaz Ur Rehman M, Farooq H, Ali MM, Ebaad Ur Rehman M, Dar QA, Hussain A. The association of subacute thyroiditis with COVID-19: a systematic review. SN Compr Clin Med. 2021 Apr 29. https://link.springer. com/10.1007/s42399-021-00912-5.

19 Lazartigues E, Qadir MMF, Mauvais-Jarvis F. Endocrine significance of SARS-CoV-2's reliance on ACE2. Endocrinology. 2020 Sep 1;161(9):bqaa108.

20 Li W, Moore MJ, Vasilieva N, Sui J, Wong SK, Berne MA, et al. Angiotensin-converting enzyme 2 is a functional receptor for the SARS coronavirus. Nature. 2003 Nov; 426(6965):450-4.

21 Wei L, Sun S, Xu CH, Zhang J, Xu Y, Zhu H, et al. Pathology of the thyroid in severe acute respiratory syndrome. Hum Pathol. 2007 Jan;38(1):95-102.

22 Zhou L, Niu Z, Jiang X, Zhang Z, Zheng Y, Wang Z, et al. SARS-CoV-2 targets by the pscRNA profiling of ACE2, TMPRSS2 and furin proteases. iScience. 2020 Nov;23(11):101744.

23 Volpé R, Row VV, Ezrin C. Circulating viral and thyroid antibodies in subacute thyroiditis. J Clin Endocrinol Metab. 1967 Sep;27(9):1275-84. 